\title{
MATRIX INTERSECTION PROBLEMS FOR CONDITIONING
}

\author{
MARKO HUHTANEN* AND OTTO SEISKARI †
}

\begin{abstract}
Conditioning of a nonsingular matrix subspace is addressed in terms of its best conditioned elements. The problem is computationally challenging. Associating with the task an intersection problem with unitary matrices leads to a more accessible approach. A resulting matrix nearness problem can be viewed to generalize the so-called Löwdin problem in quantum chemistry. For critical points in the Frobenius norm, a differential equation on the manifold of unitary matrices is derived. Another resulting matrix nearness problem allows locating points of optimality more directly, once formulated as a problem in computational algebraic geometry.
\end{abstract}

Key words. conditioning, matrix intersection problem, matrix nearness problem, Löwdin's problem, generalized eigenvalue problem

AMS subject classifications. 15A12, 65F 35

1. Introduction. This paper is concerned with the problem of conditioning of a nonsingular matrix subspace $\mathcal{V}$ of $\mathbb{C}^{n \times n}$ over $\mathbb{C}$ (or $\mathbb{R}$ ). Matrix subspaces typically appear in large scale numerical linear algebra problems where assuming additional structure is quite unrealistic. Nonsingularity means that there exists invertible elements in $\mathcal{V}$. The conditioning of $\mathcal{V}$ is then defined in terms of its best conditioned elements. In the applications that we have in mind, typically $\operatorname{dim} \mathcal{V} \ll n^{2}$. For example, in the generalized eigenvalue problem $\operatorname{dim} \mathcal{V}=2$ only. In this paper the task of assessing conditioning is formulated as a matrix intersection problem for $\mathcal{V}$ and the set of unitary matrices. ${ }^{1}$ Since this can be done in many ways, the interpretation is amenable to computations through matrix nearness problems and versatile enough in view of addressing operator theoretic problems more generally.

Denote by $U(n)$ the set of unitary matrices in $\mathbb{C}^{n \times n}$. The intersection problem for $\mathcal{V}$ and $U(n)$, which are both smooth submanifolds of $\mathbb{C}^{n \times n}$, can be formulated as a matrix nearness problem

$$
\min _{V \in \mathcal{V}, U \in U(n)}|||V-U|||
$$

in a unitarily invariant norm $\||\cdot|\|$. (Here we interpret a matrix nearness problem more generally, involving two subsets of matrices.) Other equivalent formulations can also be considered. Maximum intersection takes place with the so-called HurwitzRadon matrix subspaces [17, 24]. (See also [7].) Then, quite remarkably, any nonzero element is a scalar multiple of a unitary matrix. Whether or not the intersection is empty, the task can be viewed as a matrix nearness problem generalizing the Löwdin problem of $\operatorname{dim} \mathcal{V}=1$ [19]. See also $[9,2]$. In the operator norm the value of $(1.1)$ yields the distance to the intersection such that finding minimizers is equivalent to

* Division of Mathematics, Department of Electrical and Information Engineering, University of Oulu, 90570 Oulu 57, Finland, (Marko.Huhtanen@aalto.fi). This work has been supported by the Academy of Finland and in part by the European Commission project TODEQ (MTKD-CT-2005030042).

$\dagger$ Department of Mathematics and Systems Analysis, Aalto University, Box 1100, FIN-02015, Finland, (otto.seiskari@aalto.fi). This work has been partially supported by the Academy of Finland.

${ }^{1}$ So far, intersection problems are more common in computational geometry than in matrix analysis. This paper is concerned with quadratic surfaces (in $\mathbb{C}^{n \times n}$ ) which is a standard setting, e.g., in computer graphics [18]. 
finding a best conditioned element of $\mathcal{V}$. In the Frobenius norm a differential equation on $U(n)$ is derived for finding critical points (local minima or saddle points). This is computationally feasible by the fact that for differential equations on manifolds there are structure preserving numerical integration methods [11, p. 112]. In this paper, a simple computational scheme is derived.

Another condition for an intersection considered is

$$
\min _{V \in \mathcal{V}}\left\||| I-V^{*} V\right\| \|
$$

In the operator norm, also this problem is equivalent to finding a best conditioned element of $\mathcal{V}$. In the Frobenius norm this gives rise to a problem in computational (real) algebraic geometry when performing computations on the coefficients relative to a chosen basis of $\mathcal{V}$. This approach admits, after removing redundancy, locating local minima. As an illustration, the case $\operatorname{dim} \mathcal{V}=2$ is treated in detail.

In practice, typically in large scale problems, an improvement in conditioning of an initial guess $V \in \mathcal{V}$ may well suffice instead of finding a local minimum. For instance, in ILU preconditioning various tricks are used to improve conditioning of the factors. ${ }^{2}$ Both of these methods can be used to this end. For sparse large scale problems we regard the formulation (1.2) as more attractive.

The paper is organized as follows. In Section 2 matrix intersection problems for matrix subspaces are described. Computational methods to solve the respective nearness problems in the Frobenius norm are considered in Section 3. Section 4 is concerned with describing how conditioning of matrix subspaces arises in practice. Numerical experiments are presented in Section 5.

2. Matrix intersection problems for conditioning of matrix subspaces. Assume $\mathcal{V}$ is a matrix subspace of $\mathbb{C}^{n \times n}$ over $\mathbb{C}$ (or $\mathbb{R}$ ). Depending on whether $\mathcal{V}$ contains invertible elements, $\mathcal{V}$ is called either nonsingular or singular [15].

Definition 2.1. A matrix subspace $\mathcal{V}$ is nonsingular (singular) if the determinant function does not (does) vanish identically on $\mathcal{V}$.

Generically a matrix subspace is nonsingular; see [16] for a qualitative treatment of nonsingular matrix subspaces in view of matrix inversion. With $\operatorname{dim} \mathcal{V}=1$ we are dealing with the standard notion of nonsingularity of a matrix.

Mere nonsingularity is not sufficient for practical purposes. The standard one dimensional case is thoroughly studied. Namely, for a matrix $V \in \mathbb{C}^{n \times n}$, denote by $\sigma_{1} \geq \cdots \geq \sigma_{n} \geq 0$ its singular values and by

$$
\|V\|=\sigma_{1} \text { and }\|V\|_{F}=\sqrt{\sum_{j=1}^{n} \sigma_{j}^{2}}
$$

its operator and Frobenius norms. The condition number $\kappa(V)=\sigma_{1} / \sigma_{n}$ is employed to measure the conditioning of $V$. This scalar appears, e.g., in perturbation bounds for computing factorizations for solving linear systems and in assessing the speed of convergence of the conjugate gradient method for positive definite matrices [8].

To deal with conditioning of matrix subspaces for $\operatorname{dim} \mathcal{V} \geq 2$, it seems more natural to formulate appropriate matrix intersection problems. Classically such problems arise as follows.

\footnotetext{
${ }^{2}$ In ILU preconditioning, the matrix subspaces are defined by fixing a sparsity structure.
} 
EXAMPLE 1. Probably the most encountered matrix intersection problem is that of whether two matrix subspaces $\mathcal{V}$ and $\mathcal{W}$ intersect in the complement of the zero matrix. (Of course, both $\mathcal{V}$ and $\mathcal{W}$ contain the zero matrix. Therefore it is more natural to inspect their elements in the projective space $\mathbf{P}\left(\mathbb{C}^{n \times n}\right)$. Then the zero matrix is not accepted.) The task can be approached by comparing the singular values of the difference of the orthogonal projectors onto $\mathcal{V}$ and $\mathcal{W}$; see, for instance, $[2$, p. 201] and [8].

The most well-conditioned elements of a matrix subspace can be found by formulating matrix intersection problems with the set of unitary matrices $U(n)$ and then treat them as matrix nearness problems. (For matrix theoretic properties of $U(n)$, see [26, pp. 427-428].) Once this is realized, the problem of conditioning can be formulated in a natural way in any unitarily invariant norm.

TheOREM 2.2. Suppose $\mathcal{V}$ is a nonsingular matrix subspace of $\mathbb{C}^{n \times n}$. Then a matrix $V$ solving

$$
\min _{V \in \mathcal{V}, U \in U(n)}\|V-U\|
$$

satisfies $\kappa(V)=\frac{2}{\sigma_{n}}-1=\min _{V \in \mathcal{V}} \kappa(V)$.

Proof. Since $U(n)$ is compact, the minimum exists. As is well known, in terms of the singular values of $V \in \mathcal{V}$, the distance of $V$ to the set of unitary matrices is

$$
\max _{j=1, \ldots, n}\left|1-\sigma_{j}\right|
$$

see, e.g., [13, p.454]. Since $\mathcal{V}$ is a homogeneous set, the matrix $V$ can be scaled by a factor $t>0$ to get a matrix which is closer to $U(n)$. With the optimal scaling based on (2.2), we must have

$$
t \sigma_{1}-1=1-t \sigma_{n}
$$

i.e., $t=2 /\left(\sigma_{1}+\sigma_{n}\right)$ holds. Thereby, if $V$ solves (2.1), necessarily $t=1$ and therefore $\sigma_{1}+\sigma_{n}=2$. The condition number of $V$ is hence $\kappa(V)=2 / \sigma_{n}-1$. It remains to show that this equals $\min _{V \in \mathcal{V}} \kappa(V)$.

For this, observe first that the condition number of a matrix is scaling invariant. Thus, in the minimization problem $\min _{V \in \mathcal{V}} \kappa(V)$ the subspace $\mathcal{V}$ can be replaced with those matrices $V$ of $\mathcal{V}$ that satisfy $\sigma_{1}-1=1-\sigma_{n}$, by the above scaling argument. From this it follows that the two minimization problems have the same minimizers. $\mathrm{C}$

Corollary 2.3. If $V \in \mathcal{V}$ solves $\min _{V \in \mathcal{V}, U \in U(n)}\|V-U\|_{F}$, then the singular values of $V$ satisfy $\sum_{j=1}^{n} \sigma_{j}\left(1-\sigma_{j}\right)=0$.

Proof. For any matrix $V \in \mathcal{V}$, consider its scalings $t V$ with $t>0$. For the minimum, differentiate $\sum_{j=1}^{n}\left(1-t \sigma_{j}\right)^{2}$ and find its zeros. At an optimal matrix no scaling is needed and $t=1$ yields the condition. $\square$

We do not have a unique solution by the fact that $U(n)$ and $\mathcal{V}$ are invariant in multiplication by $e^{i \theta}$ with $\theta \in[0,2 \pi)$. (If $\mathcal{V}$ is a matrix subspace over $\mathbb{R}$, then let $\theta=0$ or $\theta=\pi$.)

It is not unconceivable that, aside from the dimension, the value of (2.1) is the single most important scalar for a matrix subspace. It is preserved under unitary equivalence; two matrix subspaces $\mathcal{V}$ and $\mathcal{W}$ are said to be unitarily equivalent if $\mathcal{W}=U_{1} \mathcal{V} U_{2}$ with $U_{1}, U_{2} \in U(n)$.

Example 2. Assume $V \in \mathbb{C}^{n \times n}$. The problem (2.1) in the one dimensional case $\mathcal{V}=\operatorname{span}\{V\}$ is equivalent to the so-called Löwdin problem in quantum chemistry 
[19]. (For more details on this and related problems, see [9, 2].) By taking the singular value decomposition $V=Q_{1} \Sigma Q_{2}^{*}$ of $V$, with $Q_{1}$ and $Q_{2}$ unitary and $\Sigma$ diagonal having nonnegative entries, the best unitary approximation to $V$ is $Q_{1} Q_{2}^{*}$.

We interpret (2.1) as a distance function to the intersection by the fact that its value expresses how much $\mathcal{V}$ should be perturbed to obtain a matrix subspace of the same dimension containing a unitary matrix. The task of finding a nearest singular matrix subspace of the same dimension to $\mathcal{V}$ is seemingly more challenging. These two extremal problems are pivotal for understanding the conditioning of matrix subspaces. Observe that there are singular matrix subspaces of dimension $O\left(n^{2}\right)$. (Take a matrix subspace with a zero column.) Thereby, even though the real dimension of $U(n)$ is as high as $n^{2}$ [23, p. 200], there exists large dimensional matrix subspaces with no intersection with $U(n)$.

According to the proof, (2.1) is a function of the extreme singular values of the elements of $\mathcal{V}$. We have

$$
\min _{V \in \mathcal{V}, U \in U(n)}\|V-U\| \leq \min _{V \in \mathcal{V}, U \in U(n)}\|V-U\|_{F} \leq \sqrt{n} \min _{V \in \mathcal{V}, U \in U(n)}\|V-U\|
$$

by standard arguments. The value of (2.1) is bounded from above by one and equals zero if and only if $\mathcal{V}$ intersects the set of unitary matrices. Its value is one if and only if $\mathcal{V}$ is a singular matrix subspace.

EXAMPLE 3. In operator space theory one is concerned with matrix subspaces $\mathcal{V}$ over $\mathbb{C}$ with the properties that $I \in \mathcal{V}$ and $V^{*} \in \mathcal{V}$ whenever $V \in \mathcal{V}$ [3, p. 47]. Such a matrix subspace is called an operator system. Hence, in operator space theory the setup is such that $\mathcal{V}$ always intersects $U(n)$. For instance, the set of Toeplitz matrices is an operator system. (Toeplitz matrices have constant diagonals.)

Maximal intersection with $U(n)$ takes place with the so-called Hurwitz-Radon matrix subspaces.

Definition 2.4. A matrix subspace $\mathcal{V}$ of $\mathbb{C}^{n \times n}$ over $\mathbb{R}$ is a Hurwitz-Radon matrix subspace if each of its nonzero elements is a scalar multiple of a unitary matrix. ${ }^{3}$

The scalar

$$
\min _{V \in \mathcal{V}} \kappa(V)
$$

is a natural candidate for measuring the conditioning of a matrix subspace $\mathcal{V}$. (For measures based on the use of the determinant, see [15].) Its computation seems very challenging, though. The equivalent interpretation (2.1) is more accessible since there are several equivalent ways to formulate conditions for an intersection. (It is, moreover, versatile enough allowing to consider, for example, other classical matrix groups in place of $U(n)$.) In terms of a unitarily invariant norm $|\|\cdot \mid\|$, it is natural to consider

$$
\min _{V \in \mathcal{V}}\left\|\mid I-V^{*} V\right\| \|
$$

By involving a single matrix, the identity, and a subset of $\mathbb{C}^{n \times n}$, this formulation resembles more a typical matrix nearness problem. (Therefore, unlike (1.1), this is nontrivial only for $\operatorname{dim} \mathcal{V}>1$.) We have a nonnegative scalar equaling zero if and only if $\mathcal{V}$ contains a unitary matrix. Moreover,

$$
\min _{V \in \mathcal{V}}\left\|I-V^{*} V\right\| \leq \min _{V \in \mathcal{V}}\left\|I-V^{*} V\right\|_{F} \leq \sqrt{n} \min _{V \in \mathcal{V}}\left\|I-V^{*} V\right\|
$$

\footnotetext{
${ }^{3}$ Any Hurwitz-Radon matrix subspace of dimension two at least is necessarily a subspace over $\mathbb{R}$.
} 
holds. In the operator norm this matrix nearness problem is also equivalent to determining $\min _{V \in \mathcal{V}} \kappa(V)$ as follows.

TheOREM 2.5. Suppose $\mathcal{V}$ is a nonsingular matrix subspace of $\mathbb{C}^{n \times n}$. Then a matrix $V$ solving

$$
\min _{V \in \mathcal{V}}\left\|I-V^{*} V\right\|
$$

satisfies $\kappa(V)=\sqrt{\frac{2}{\sigma_{n}^{2}}-1}=\min _{V \in \mathcal{V}} \kappa(V)$.

Proof. For any element $V \in \mathcal{V}$, consider its scalings $t V$ with $t>0$. By using (2.2), $\mathrm{nn}$ optimal value for the scaling $t$ to minimize $\left\|I-t^{2} V^{*} V\right\|$ in the operator norm satisfies

$$
t^{2} \sigma_{1}^{2}-1=1-t^{2} \sigma_{n}^{2} .
$$

Consequently, $t=\sqrt{2} /\left(\sigma_{1}^{2}+\sigma_{n}^{2}\right)^{1 / 2}$. At an optimal matrix $V$, necessarily $t=1$ and thereby $\sigma_{1}^{2}+\sigma_{n}^{2}=2$. The condition number of $V$ is hence $\kappa(V)=\sqrt{2-\sigma_{n}^{2}} / \sigma_{n}$. It remains to show that this equals $\min _{V \in \mathcal{V}} \kappa(V)$.

For this, combine the arguments of the proof of Theorem 2.2 with the fact that the map $t \rightarrow t^{2}$ is increasing for $t>0$.

The claim concerning the Frobenius norm can be proved similarly.

Corollary 2.6. If $V \in \mathcal{V}$ solves $\min _{V \in \mathcal{V}}\left\|I-V^{*} V\right\|_{F}$, then the singular values of $V$ satisfy $\sum_{j=1}^{n} \sigma_{j}^{2}\left(1-\sigma_{j}^{2}\right)=0$.

Proof. For any matrix $V \in \mathcal{V}$, consider its scalings $t V$ with $t>0$. For the minimum, differentiate $\sum_{j=1}^{n}\left(1-t^{2} \sigma_{j}^{2}\right)^{2}$ and find its zeros. At an optimal matrix no scaling is needed and $t=1$ yields the condition.

Compared with Corollary 2.3, now the singular values are weighed more nonsymmetrically around one as can be seen by the factoring a single term

$$
\sigma_{j}^{2}\left(1-\sigma_{j}^{2}\right)=\sigma_{j}\left(1+\sigma_{j}\right) \sigma_{j}\left(1-\sigma_{j}\right)
$$

in the latter sum.

To end this section, the measures proposed yield incomplete overall information on the conditioning of a matrix subspace. To illustrate this, take a singular matrix subspace and perturb just one of its elements to have a nonsingular matrix subspace. (A related problem: for a nonsingular matrix subspace, find its singular subspace of the largest possible dimension.) None of the measures reflect the fact that the nonsingularity is a result of such a minor modification of a singular matrix subspace. An apparent problem is that, at present, we do not have an analogy of the singular value decomposition for matrix subspaces.

3. Computing local minima for the nearness problems. Next we are concerned with finding local minima for the above minimization problems in the Frobenius norm. Here the standard inner product

$$
(A, B)=\operatorname{trace}\left(B^{*} A\right)
$$

on $\mathbb{C}^{n \times n}$ is used. For matrix subspaces over $\mathbb{R}$, take the real part and denote the respective inner product by $(A, B)_{\mathbb{R}}=\operatorname{Re}(A, B)$. 
3.1. Flow on the manifold of unitary matrices. For the operator norm $(2.1)$ is apparently not readily computed. For a matrix subspace $\mathcal{V}$ of $\mathbb{C}^{n \times n}$ over $\mathbb{C}$ (or $\mathbb{R}$ ), consider the matrix nearness problem

$$
\min _{V \in \mathcal{V}, U \in U(n)}\|V-U\|_{F}
$$

instead. Of course, these problems are related through (2.4). By regarding the set of unitary matrices as a smooth Riemannian submanifold of $\mathbb{C}^{n \times n}$, a differential equation is derived for locating critical points. ${ }^{4}$ Because of the structure of $U(n)$, in what follows, we regard $\mathcal{V}$ as a vector space over $\mathbb{R}$.

EXAmple 4. Bear in mind that there can exist many local minima. For example, take $\mathcal{V}$ to be the set of Toeplitz matrices. Then $\mathcal{V}$ contains the identity matrix. For other unitary matrices, take a Toeplitz matrix having ones on the first subdiagonal and $e^{i \theta}$ at the $(1, n)$ position with any $\theta \in \mathbb{R}$.

For a differential equation, we reason geometrically to find a unitary matrix corresponding to a local minimum or a saddle point of the problem (3.2). First decompose any given unitary matrix $Q$ as

$$
Q=V+V_{\perp}
$$

with $V \in \mathcal{V}$ and $V_{\perp}$ in the orthogonal complement of $\mathcal{V}$. For a necessary condition on $Q$ to solve (3.2), the orthogonal part $V_{\perp}$ must be orthogonal to the tangent space $\mathrm{T}_{Q}(U(n))$ of $U(n)$ at $Q$. (Hence, if $Q$ solves (3.2), take $V$ according to (3.3).)

To enforce orthogonality, in (3.3) decompose the orthogonal part $V_{\perp}$ further as

$$
V_{\perp}=V_{\mathrm{T}}+V_{\mathrm{T} \perp}
$$

with $V_{\mathrm{T}} \in \mathrm{T}_{Q}(U(n))$ and $V_{\mathrm{T} \perp}$ in the orthogonal complement of $\mathrm{T}_{Q}(U(n))$; see Figure 3.1. Then define a smooth vector field on $U(n)$ as

$$
-V_{\mathrm{T}}
$$

at $Q$. There holds $\left(V,-V_{T}\right)_{\mathbb{R}}=\left\|V_{T}\right\|^{2}$, so that the component in $\mathcal{V}$ increases along the flow. We have a critical point if and only if $V_{\perp}=V_{\mathrm{T} \perp}$, i.e., the orthogonal part $V_{\perp}$ is orthogonal to the tangent space.

Consider the flow generated by the vector field (3.4). To reach a minimum from an arbitrary starting point $Q$, the connectedness of $U(n)$ is a prerequisite for the success of this approach. (Of course $U(n) \subset\left\{M \in \mathbb{C}^{n \times n}:\|M\|_{F}=\sqrt{n}\right\}$.) Recall though that the set of unitary matrices is not simply connected; its fundamental group is $\mathbb{Z}$.

Aside from connectedness, to form the vector field (3.4) in practice, the tangent spaces of $U(n)$ must be computable. Typically they are derived by considering curves $a(t)$ satisfying $a(0)=Q \in U(n)$ and $a(t)^{*} a(t)=I$ for small $|t| \geq 0$. Then, after differentiating, one can conclude that at $Q$ the tangent space is

$$
\left\{Q i H: \text { with } H^{*}=H\right\}
$$

which is a vector space over $\mathbb{R}$. Thereby the vector field (3.4) can be given as

$$
-V_{\mathrm{T}}=-\frac{1}{2} Q\left(Q^{*} V_{\perp}-V_{\perp}^{*} Q\right)=-\frac{1}{2}\left(V_{\perp}-Q V_{\perp}^{*} Q\right)
$$

\footnotetext{
${ }^{4}$ The approach presented can be taken with $U(n)$ replaced with any smooth connected submanifold of $\mathbb{C}^{n \times n}$ whose tangent spaces can be readily computed and projection back to the manifold is feasible. (For instance, symplectic matrices constitute another classical matrix group of importance.)
} 


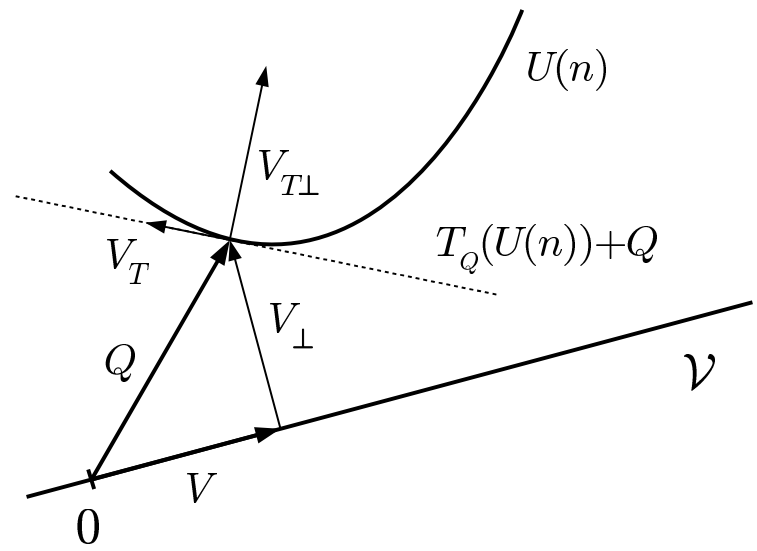

FIG. 3.1. Decomposing $Q$ as $Q=V+V_{\perp}$ and then $V_{\perp}$ as $V_{\perp}=V_{T}+V_{T \perp}$.

which is an $O\left(n^{3}\right)$ computation at most once $V_{\perp}$ has been computed. Observe that we have a critical point if and only if $Q^{*} V_{\perp}$ is Hermitian. Instead of trying to use this fact in solving the corresponding nonlinear equations, we proceed by solving for the flow.

There are several numerical integration methods for solving differential equations on manifolds [11]. Because of its central role in applications, the unitary case has received attention early [6]. For an inexpensive structure preserving numerical integration method, set $i H=-\frac{1}{2}\left(Q^{*} V_{\perp}-V_{\perp}^{*} Q\right)$. Certainly, $\|H\|_{F}=\left\|V_{\mathrm{T}}\right\|_{F} \leq\|Q\|_{F}=\sqrt{n}$. Then define

$$
Q\left(I-\frac{\triangle t}{2} i H\right)^{-1}\left(I+\frac{\triangle t}{2} i H\right) .
$$

Based on the use of the Cayley transform, this is unitary satisfying

$$
Q\left(I-\frac{\triangle t}{2} i H\right)^{-1}\left(I+\frac{\triangle t}{2} i H\right) \approx Q+\triangle t Q i H+O\left(h^{2} H^{2}\right)
$$

by using the Neumann series with $\left(I-\frac{\Delta t}{2} i H\right)^{-1}$. Observe that $Q i H=-V_{\mathrm{T}}$, so that the first order term is in the direction of the vector field. The method (3.7) can be seen as a linearly implicit Runge-Kutta method [10, p. 102]. Regarding the choice of the step-length, we use standard methods.

Once a critical point (local minimum or saddle point) is reached, we have $Q$ and $V$ satisfying

$$
Q=V+V_{\perp}=V+V_{T \perp} .
$$

To improve this, compute the nearest unitary matrix $\hat{Q}$ to $V$. If $\hat{Q} \neq Q$, compute the trajectory starting from $\hat{Q}$.

EXAMPLE 5. Although most likely a curiosity, consider computing the unitary polar factor of $M \in \mathbb{C}^{n \times n}$ with the flow. Assume $\|M\|_{F}=1$. For this, look at the one dimensional case $\mathcal{V}=\operatorname{span}_{\mathbb{R}}\{M\}$. Then $V_{\perp}=Q-(Q, M)_{\mathbb{R}} M$ and

$$
i H=\frac{-(Q, M)_{\mathbb{R}}}{2}\left(Q^{*} M-M^{*} Q\right) .
$$

(For methods to compute the polar factor, see [8]. Note that in the iterative methods for the polar factor, the iterates are not unitary.) Poor starting points may not lead 
to the polar factor. For example, if $M$ is Hermitian, then the starting point $Q=I$ is a critical point. (This is certainly an unfortunate initial guess. A multiple $e^{i \theta} I$ of the identity is now a critical point only for $\theta=0, \pi / 2, \pi$ or $3 \pi / 2$.)

For more advanced structure preserving numerical integration methods on $U(n)$, projection back to the manifold must be feasible. With $U(n)$ the projection of a matrix $M \in \mathbb{C}^{n \times n}$ is accomplished with the singular value decomposition $M=Q_{1} \Sigma Q_{2}^{*}$ as described in Example 2. The nearest unitary matrix to $M$ is $Q_{1} Q_{2}^{*}$, i.e., the unitary polar factor of $M$. Observe that the time step (3.7) is devised in such a way that this projection is superfluous.

Classifying the critical points computed does not appear to be simple. With respect to the condition (2.6) in the Frobenius norm this can be done more readily.

3.2. Direct optimality conditions. For finding (2.6), consider formulating computable conditions for optimality in the Frobenius norm. As opposed to (3.2), now the matrix subset

$$
\left\{V^{*} V: V \in \mathcal{V}\right\}
$$

involved is not readily characterizable, except that it is closed and homogeneous over $\mathbb{R}$. This leads us to perform computations on the coefficients relative to a fixed basis $V_{1}, \ldots, V_{k}$ of the matrix subspace $\mathcal{V}$ over $\mathbb{C}$. (Proceed similarly with subspaces over $\mathbb{R}$.) As a result, a system of polynomial equations is obtained. Hence, we are faced with a problem in computational algebraic geometry.

In terms of the basis, define

$$
H\left(z_{1}, \ldots, z_{k}\right)=\left(z_{1} V_{1}+\cdots+z_{k} V_{k}\right)^{*}\left(z_{1} V_{1}+\cdots+z_{k} V_{k}\right)
$$

from $\mathbb{C}^{k}$ to the set of $n$-by- $n$ Hermitian matrices $\mathcal{H}$. Since $\mathcal{H}$ is a matrix subspace of $\mathbb{C}^{n \times n}$ over $\mathbb{R}$, the real part of the inner product (3.1) is used. Identifying $\mathbb{C}^{k}$ with $\mathbb{R}^{2 k}$, from now on we regard $\mathcal{V}$ as a vector space over $\mathbb{R}$. Then we are dealing with a smooth map (3.8) whose rank is $2 k-1$ at most at any point. (For the rank of a smooth map at a point, see, e.g., [23, p. 228].)

Proposition 3.1. Suppose $\mathcal{V}$ is a matrix subspace of $\mathbb{C}^{n \times n}$ over $\mathbb{C}$ of dimension $k$. When regarded as a subspace over $\mathbb{R}$, at any point the rank of the map (3.8) is at most $2 k-1$.

Proof. Fix $V, V_{0} \in \mathcal{V}$. Then linearize the curve $t \mapsto\left(V+t V_{0}\right)^{*}\left(V+t V_{0}\right)$, with $t \in \mathbb{R}$, to have the linear map $V_{0} \mapsto V^{*} V_{0}+V_{0}^{*} V$ from $\mathcal{V}$ to $\mathcal{H}$. Its rank yields the rank of (3.8) at $V$. It is at most $2 k-1$ since its nullspace contains $i V_{0}$.

As a consequence, looking for the critical points of the function

$$
\left(z_{1}, \ldots, z_{k}\right) \longmapsto\left\|I-H\left(z_{1}, \ldots, z_{k}\right)\right\|_{F}^{2}
$$

to find a local minimum is not advisable by the fact that we have too many degrees of freedom. To remove a redundant degree of freedom, observe that

$$
H\left(t e^{i \theta} z_{1}, \ldots, t e^{i \theta} z_{k}\right)=t^{2} H\left(z_{1}, \ldots, z_{k}\right)
$$

for any $t \in \mathbb{R}$ and $\theta \in[0,2 \pi)$. By using this, we may impose $\theta$ to be chosen in such a way that $z_{2}$ is kept real and then look for the critical points.

We proceed more geometrically to save storage and reduce computational cost with sparse problems. Clearly, there is a unitary matrix in $\mathcal{V}$ if and only if the image of the map (3.8) contains the identity. Otherwise, to find a matrix $H=H\left(z_{1}, \ldots, z_{k}\right)$ 
in the image nearest to the identity, the difference $I-H$ must be orthogonal to the range of the linearization of (3.8) at $H$. This happens exactly at the critical points of (3.9). To locate them geometrically, set

$$
H_{j k}=\frac{1}{2}\left(V_{j}^{*} V_{k}+V_{k}^{*} V_{j}\right) \text { and } K_{j k}=\frac{1}{2 i}\left(V_{j}^{*} V_{k}-V_{k}^{*} V_{j}\right)
$$

for $j \leq k$. Denote $z_{j}=x_{j}+i y_{j}$ for $j=1, \ldots, k$. (If the basis matrices $V_{j}$ are orthogonal against one another, then all but $H_{j j}$ are trace zero matrices. Choosing the basis orthonormal may be advisable for numerical stability.) Then the respective element in the image of (3.8) can be written as

$$
\sum_{j=1}^{k}\left(x_{j}^{2}+y_{j}^{2}\right) H_{j j}+2 \sum_{j=1}^{k-1} \sum_{k>j}\left(\left(x_{j} x_{k}+y_{j} y_{k}\right) H_{j k}-\left(x_{j} y_{k}-y_{j} x_{k}\right) K_{j k}\right) .
$$

Linearizing this yields a basis of the range of the linearization of (3.8) at $H$.

The two dimensional case $k=2$ is illustrative and thereby treated in detail in the following proposition. In practice, the two dimensional case appears in the generalized eigenvalue problem.

By Proposition 3.1, in the two dimensional case the rank of the map (3.8) is at most three. It suffices to consider the case of $z_{2}$ being real.

Proposition 3.2. Assume $k=2$. For $x_{1} \neq 0$ and $x_{2} \neq 0$, the range of the linearization of (3.8) at $\left(x_{1}, y_{1}, x_{2}, 0\right)$ is spanned by $M_{1}=x_{1} H_{11}+x_{2} H_{12}, M_{2}=$ $x_{2} H_{22}+x_{1} H_{12}+y_{1} K_{12}$ and $M_{3}=y_{1} H_{12}-x_{1} K_{12}$.

Proof. At $\left(x_{1}, x_{2}, y_{1}, y_{2}\right)$ the linearization has the image

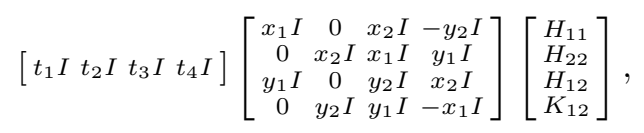

where the coefficients $t_{j} \in \mathbb{R}, j=1, \ldots, 4$ can be chosen freely. (The zero blocks are of size $n$-by- $n$.) Compute the LU factorization of the appearing 4-by-4 block matrix. If $x_{1} \neq 0$ and $x_{2} \neq 0$, then the $U$ factor reads

$$
\left[\begin{array}{cccc}
x_{1} I & 0 & x_{2} I & -y_{2} I \\
0 & x_{2} I & x_{1} I & y_{1} I \\
0 & 0 & \left(x_{1} y_{2}-x_{2} y_{1}\right) / x_{1} I & \left(x_{1} x_{2}+y_{1} y_{2}\right) / x_{1} I \\
0 & 0 & 0
\end{array}\right]
$$

proving the claim once $y_{2}$ is set to zero and $-x_{2} / x_{1}$ is taken as a common factor in the third row.

It is immediate that if the matrices $H_{11}, H_{12}, H_{22}$ and $K_{12}$ are linearly independent, then the spanning matrices of Proposition 3.2 are linearly independent. Imposing orthogonality against

$$
I-H\left(x_{1}, y_{1}, x_{2}, 0\right)=I-\left(x_{1}^{2}+y_{1}^{2}\right) H_{11}-x_{2}^{2} H_{22}-2 x_{2}\left(x_{1} H_{12}+y_{1} K_{12}\right)
$$

leads to three polynomial equations of degree three in the variables $x_{1}, y_{1}$ and $x_{2}$. It is noteworthy that in large scale problems the spanning matrices $V_{1}$ and $V_{2}$ are often sparse. Then, performing computations in sparse-sparse mode, it is not inconceivable to deal with the matrices $H_{11}, H_{12}, H_{22}$ and $K_{12}$ in realistic applications to compute the traces needed. (Being Hermitian, only half of the nonzero entries are stored. Hence, in reality, only two sparse matrices need to be stored.) For operations in sparse mode, see [25, Chapter 10]. 
Whether the number of solutions to the system of polynomial equations obtained is finite can be inspected by invoking methods from computational algebraic geometry [5, Chapter 2]. If so, then to estimate their number, there are Bézout's and Bernstein's theorems [5]. These are pessimistic estimates since we are interested in real solutions only. For the real case, see [22].

To check whether a critical point computed is a local minimum, one can inspect the eigenvalues of the Hessian of (3.9) with the restriction $z_{2}=x_{2}$. Of course, with the computed critical points, it is less expensive just to compare the values of (3.9).

In case $\operatorname{dim} \mathcal{V}>2$ these two dimensional techniques can also be used to improve the conditioning of an initial guess $V_{1} \in \mathcal{V}$ with a "descend" direction $V_{2} \in \mathcal{V}$. This can be repeated to have a descend method.

4. Conditioning in applications. Cases in which the conditioning of a matrix subspace is of interest are (non exhaustively) described in the examples that follow. Matrix subspaces typically appear in large scale numerical linear algebra problems where assuming additional structure is unrealistic.

4.1. The generalized eigenvalue problem. A matrix subspace problem arising in connection with the generalized eigenvalue problem involves the invertibility of the elements of a nonsingular matrix subspace $\mathcal{V}=\operatorname{span}\left\{V_{1}, V_{2}\right\}$ with $V_{1}, V_{2} \in \mathbb{C}^{n \times n}$. (For the generalized eigenvalue problem, see [21].) In large scale applications these spanning matrices are typically also sparse.

The problem is concerned with transforming the generalized eigenvalue problem

$$
V_{1} x=\lambda V_{2} x
$$

into the standard eigenvalue problem. Then it is of interest to have a fixed element $V=z_{1} V_{1}+z_{2} V_{2}$ of $\mathcal{V}$ that is as well-conditioned as possible in considering the formulation

$$
V_{1} y=\mu\left(z_{1} V_{1}+z_{2} V_{2}\right) y
$$

equivalent to (4.1). (Now multiply with the inverse of $V$ from the left.) This problem is mentioned (in passing) in the positive definite case in [21, p. 344].

4.2. Matrix factorization problems. Let $A \in \mathbb{C}^{n \times n}$ and assume $\mathcal{W}$ and $\mathcal{V}_{1}$ are matrix subspaces of $\mathbb{C}^{n \times n}$ over $\mathbb{C}($ or $\mathbb{R})$. With $W \in \mathcal{W}$ consider the map

$$
W \longmapsto\left(I-P_{1}\right) A W
$$

where $P_{1}$ is a projection on $\mathbb{C}^{n \times n}$ onto $\mathcal{V}_{1}$ and $I$ denotes the identity matrix (regarded as acting on $\mathbb{C}^{n \times n}$ ). If this linear map has a nontrivial nullspace possessing invertible elements $W$, then

$$
A=V_{1} W^{-1}
$$

with $V_{1}=P_{1} A W$. (The dimension of the nullspace measures how nonunique the factorization is.) This is a very general method to compute factorizations; for more details, see $[14,15]$. Denote by $\mathcal{V}$ the nullspace of $(4.2)$.

For two reasons, in practice the factorization (4.3) is not exact. First, because of finite precision, the computation of the nullspace is always approximative. Second, in very large scale problems exact factoring is not even realistic. Then the dimensions 
of $\mathcal{W}$ and $\mathcal{V}_{1}$ are very moderate compared with $n^{2}$ and thereby an arbitrary matrix can be factored only approximately [4]. The factors computed do satisfy

$$
\frac{\left\|A-V_{1} W^{-1}\right\|}{\left\|W^{-1}\right\|} \leq\left\|A W-V_{1}\right\| \leq\left\|A-V_{1} W^{-1}\right\|\|W\|
$$

in the operator norm. How accurately (4.3) can be expected to hold is hence related with the conditioning of the nullspace $\mathcal{V}$ (or an approximate nullspace).

Classical factorizations are rarely unique. The available degrees of freedom should be used to choose well-conditioned factors.

EXAmPle 6. Gaussian elimination is a matrix factorization result ${ }^{5}$, expressed in terms of (and occasionally overshadowed by) an algorithm. In computing an LU factorization of a matrix $A \in \mathbb{C}^{n \times n}$, one deals with the matrix subspace $\mathcal{V}=L \mathcal{D}$, where $L$ is an invertible lower triangular matrix and $\mathcal{D}$ denotes the set of diagonal matrices. Hence, $\operatorname{dim} \mathcal{V}=n$. (If partial pivoting is used, replace $A$ with $P A$, where $P$ is a permutation.) Typically $V \in \mathcal{V}$ with unit diagonal is chosen in practice, although it is not necessarily an optimal element in view of the criteria proposed. With $n=2$ simple examples can be devised to see this. Of course, only if $\mathcal{V}=\mathcal{D}$ there is a unitary element in $\mathcal{V}$.

EXAMPLE 7. In representing $A \in \mathbb{C}^{n \times n}$ as the product of two symmetric matrices, the dimension of the nullspace $\mathcal{V}$ is least $n$ [14]. (This is a classical factorization [12].) Aside from an interest to have a well-conditioned element, a nonempty intersection with unitary matrices would give rise to a factorization of $A$ as the product of a symmetric and a symmetric unitary matrix.

4.3. Approximating with a unitary matrix in experiments. So-called scattering parameters describe the electrical behavior of a linear electrical network. (See, for example, [20].) Linearity means that the underlying operation involves a matrix. A network analyzer is an instrument aimed at measuring the entries of such a matrix. Since there can be variations in measurements, consider repeating these experiments.

With lossless networks one deals with unitary matrices. Thus, after $k \ll n$ experiments, suppose we are given matrices $V_{1}, \ldots, V_{k}$ that should all equal the same unitary matrix $Q$. Due to errors in measurements, none of the matrices is exactly unitary. How should one choose the best unitary approximation now?

In the case $k=1$ the task reduces to the Löwdin problem. For $k>1$ we propose taking $\mathcal{V}$ to be the span of the measurement matrices $V_{1}, \ldots, V_{k}$ and then solving a nearness problem considered in Section 3. The resulting unitary matrix $U$ is then taken to be an approximation to $Q$. Thus, unlike in the problems described earlier in this section, now we are not looking for an element of $\mathcal{V}$ but a unitary matrix that is closest to $\mathcal{V}$.

5. Numerical experiments. Next numerical experiments are described in the two dimensional case. The method of Section 3.2 is considered before that of Section 3.1. The reason for this change of order is computational. It is more economical (and hence, more of practical interest) to perform computations on the coefficients relative to a chosen basis of $\mathcal{V}$.

\footnotetext{
${ }^{5} A \in \mathbb{C}^{n \times n}$ has an LU factorization if and only if $A$ is strongly nonsingular.
} 
5.1. Direct optimality conditions. In the two dimensional case, to find a wellconditioned element of $\mathcal{V}=\operatorname{span}\left\{V_{1}, V_{2}\right\}$ we consider (2.6) in the Frobenius norm. As described in Section 3.2, this leads us to look for

$$
V=V\left(x_{1}, y_{1}, x_{2}\right)=\left(x_{1}+y_{1} i\right) V_{1}+x_{2} V_{2}
$$

with $x_{1}, y_{1}, x_{2} \in \mathbb{R}$ satisfying

$$
\left(I-V^{*} V, M_{1}\right)_{\mathbb{R}}=\left(I-V^{*} V, M_{2}\right)_{\mathbb{R}}=\left(I-V^{*} V, M_{3}\right)_{\mathbb{R}}=0,
$$

where the spanning matrices of Proposition 3.2 are

$$
\begin{aligned}
& M_{1}=x_{1} V_{1}^{*} V_{1}+\frac{x_{2}}{2}\left(V_{1}^{*} V_{2}+V_{2}^{*} V_{1}\right) \\
& M_{2}=\frac{-i y_{1}}{2}\left(V_{1}^{*} V_{2}-V_{2}^{*} V_{1}\right)+\frac{x_{1}}{2}\left(V_{1}^{*} V_{2}+V_{2}^{*} V_{1}\right)+x_{2} V_{2}^{*} V_{2}, \\
& M_{3}=\frac{i x_{1}}{2}\left(V_{1}^{*} V_{2}-V_{2}^{*} V_{1}\right)+\frac{y_{1}}{2}\left(V_{1}^{*} V_{2}+V_{2}^{*} V_{1}\right)
\end{aligned}
$$

The polynomial system (5.2) can be solved seemingly robustly with the existing specialized software. We used a freely available software called BERTINI [1] which is based on homotopy continuation. We also experimented with commercial symbolic software to find closed form solutions. This did not seem very attractive by the fact that the CPU time required to solve the polynomial system was somewhat unpredictable.

Regarding the tests, two small dimensional examples are described first. Then a more realistic example is concerned with a generalized eigenvalue problem taken from Matrix Market collection.

EXAMPLE 8. In this example we have

$V_{1}=\left[\begin{array}{cccc}0 & 2 & 0 & 0 \\ 0 & 1 & 1-i & 1 \\ -1+i & -1 & -i & -2 \\ -1+i & -2 & -1-2 i & -1\end{array}\right]$ and $V_{2}=\left[\begin{array}{cccc}-1 & 2-i & i & i \\ 0 & -1+3 i & 1-2 i & -2+i \\ i & 1+i & -1-i & i \\ 0 & 1+i & -i & 1-i\end{array}\right]$

These spanning matrices of $\mathcal{V}$ lead to a polynomial system with integer coefficients. See Table 5.1 for the critical points and conditioning. (The symbolic software MATHEMATICA managed also to solve the problem in less than a minute.) We had four different critical points (modulo multiplying by -1 ).

ExAMPLE 9. In this simple example we take $V_{1}=I+\tilde{V}$ and $V_{2}=I-\tilde{V}$, where

$$
\tilde{V}=\left[\begin{array}{cccc}
\frac{49}{100}+\frac{13 i}{50} & \frac{3}{2}+\frac{13 i}{100} & -\frac{21}{50}-\frac{3 i}{200} & \frac{21}{1000}+\frac{49 i}{500} \\
-\frac{7}{20}+\frac{41 i}{100} & \frac{18}{25}-\frac{9 i}{25} & \frac{29}{50}-\frac{6 i}{5} & \frac{14}{25}+\frac{43 i}{100} \\
-\frac{6}{5}+\frac{69 i}{100} & -\frac{13}{10}-\frac{9 i}{50} & -\frac{57}{1000}-\frac{3 i}{2} & -\frac{12}{5}+\frac{23 i}{100} \\
-1+\frac{18 i}{25} & -\frac{9}{5}+\frac{47 i}{100} & -\frac{11}{10}-\frac{3 i}{2} & -1-\frac{27 i}{100}
\end{array}\right]
$$

Now the matrix subspace $\mathcal{V}$ clearly contains a unitary element (all the multiples of the identity matrix) and the purpose of this test is to show that we are able to locate it. See Table 5.2 for the critical points and conditioning. (MathematicA was also able to solve the problem symbolically in less than a minute.) We had five different critical points (modulo multiplying by -1 ). 
TABLE 5.1

Critical points and conditioning of Example 8.

\begin{tabular}{rrr|cc}
$x_{1}$ & $y_{1}$ & $x_{2}$ & $\left\|I-V^{*} V\right\|_{F}$ & $\kappa(V)$ \\
\hline 0.184 & 0.144 & 0.148 & 1.246 & 19.57 \\
-0.065 & -0.071 & 0.183 & 1.554 & 17.554 \\
0.206 & -0.029 & -0.098 & 1.611 & 8.521 \\
-0.032 & -0.06 & 0.189 & 1.554 & 11.204 \\
0.032 & 0.06 & -0.189 & 1.554 & 11.204 \\
-0.184 & -0.144 & -0.148 & 1.246 & 19.57 \\
-0.206 & 0.029 & 0.098 & 1.611 & 8.521 \\
0.065 & 0.071 & -0.183 & 1.554 & 17.554
\end{tabular}

TABLE 5.2

Critical points and conditioning of Example 9.

\begin{tabular}{rrr|cc}
$x_{1}$ & $y_{1}$ & $x_{2}$ & $\left\|I-V^{*} V\right\|_{F}$ & $\kappa(V)$ \\
\hline 0.5 & 0 & 0.5 & 0 & 1 \\
-0.003 & -0.018 & -0.215 & 1.597 & 14.581 \\
0 & 0.015 & -0.218 & 1.593 & 21.07 \\
0.003 & 0.018 & 0.215 & 1.597 & 14.581 \\
0 & -0.015 & 0.218 & 1.593 & 21.07 \\
-0.5 & 0 & -0.5 & 0 & 1 \\
0 & -0.179 & 0.171 & 1.475 & 34.035 \\
0 & 0.2 & 0.097 & 1.437 & 5.163 \\
0 & -0.2 & -0.097 & 1.437 & 5.163 \\
0 & 0.179 & -0.171 & 1.475 & 34.035
\end{tabular}

EXAMPLE 10. In our third numerical experiment we have

$$
V_{1}=\frac{\tilde{V}_{1}}{\left\|\tilde{V}_{1}\right\|_{F}} \text { and } V_{2}=\frac{\tilde{V}_{2}-\left(\tilde{V}_{2}, V_{1}\right) V_{1}}{\left\|\tilde{V}_{2}-\left(\tilde{V}_{2}, V_{1}\right) V_{1}\right\|_{F}}
$$

forming an orthonormal basis of $\mathcal{V}$. Here $\tilde{V}_{1}, \tilde{V}_{2} \in \mathbb{C}^{961 \times 961}$ are the matrices DWG961A and DWG961B from Matrix Market appearing in a generalized eigenvalue problem $\tilde{V}_{1} x=\lambda \tilde{V}_{2} x$. (In realistic problems, the original spanning matrices may differ significantly, e.g., in norm. It seems advisable to replace them with an orthonormal basis.) The condition number of $V_{2}$ in this case is approximately $1.12 \cdot 10^{7}$ and $V_{1}$ is singular. See Table 5.3 for the critical points and conditioning. We had six different critical points (modulo multiplying by -1 ) such that elements with condition number of order $10^{5}$ were found.

5.2. Numerical integration on the unitary matrices. The method derived in Section 3.1 is formulated as Algorithm 1 by using a random unitary matrix as a starting point. Regarding the numerical experiments, Algorithm 1 always converged, although the number of iterations needed to reach a local minimum with a given tolerance $\epsilon$ varied with problem dimension $n$, the specific problem and the starting point $Q$. Problems with greater $n$ seemed to have multiple local minima more often and the variance in the number of iterations needed to reach them from different starting points $Q$ also seemed to increase with $n$.

Regarding the dominating operations of Algorithm 1, in addition to forming $V_{\perp}$, 
TABLE 5.3

Critical points and conditioning of Example 10.

\begin{tabular}{rrr|cc}
$x_{1}$ & $y_{1}$ & $x_{2}$ & $\left\|I-V^{*} V\right\|_{F}$ & $\kappa(V)$ \\
\hline 6.763 & 2.649 & 5.674 & 29.594 & $1.0499 \cdot 10^{5}$ \\
6.578 & 2.881 & -5.685 & 29.619 & $8.4919 \cdot 10^{4}$ \\
6.721 & 2.663 & -0.054 & 30.144 & $1.7400 \cdot 10^{6}$ \\
-6.578 & -2.881 & 5.685 & 29.619 & $8.4919 \cdot 10^{4}$ \\
-6.763 & -2.649 & -5.674 & 29.594 & $1.0499 \cdot 10^{5}$ \\
-6.721 & -2.663 & 0.054 & 30.144 & $1.7400 \cdot 10^{6}$ \\
0 & 4.601 & 5.690 & 29.908 & $1.0964 \cdot 10^{5}$ \\
0.016 & -0.031 & 5.691 & 30.473 & $3.4739 \cdot 10^{6}$ \\
0 & -4.623 & 5.682 & 29.918 & $9.5620 \cdot 10^{4}$ \\
0 & 4.623 & -5.682 & 29.918 & $9.5620 \cdot 10^{4}$ \\
-0.016 & 0.031 & -5.691 & 30.473 & $3.4739 \cdot 10^{6}$ \\
0 & -4.601 & -5.690 & 29.908 & $1.0964 \cdot 10^{5}$
\end{tabular}

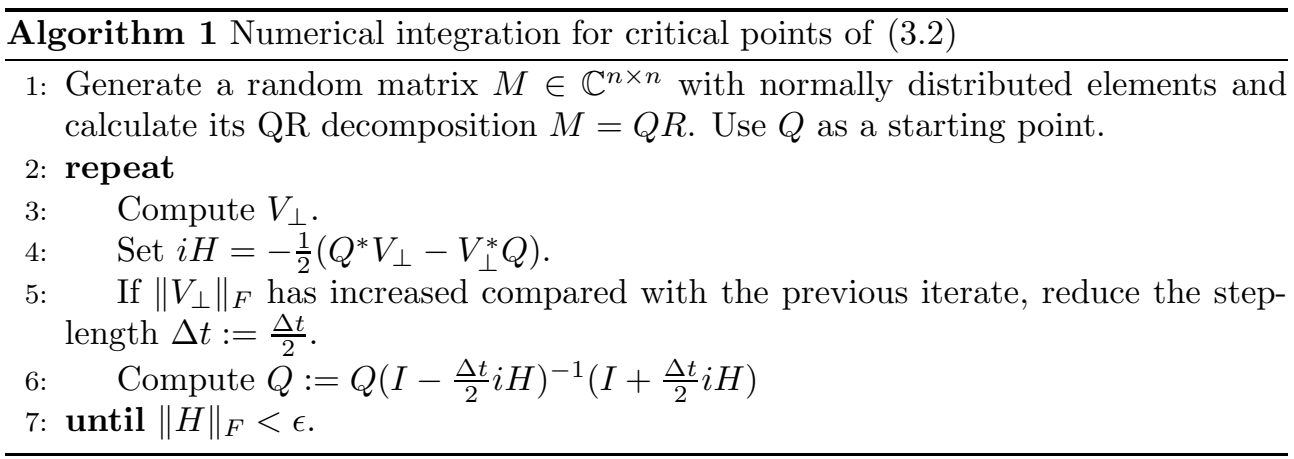

we need to perform two matrix-matrix products and perform one matrix inversion per repeat.

EXAMPLE 11. In this example the matrix subspace is that of Example 8. Now the problem (3.2) seems to have a unique solution (modulo multiplication by $e^{i \theta}$ ). It was reached with $\epsilon=10^{-3}$ and $\Delta t_{0}=1$ after $70-80$ iterations, on the average. Then $\kappa(V) \approx 9.7$.

EXAmPle 12. In this example the matrix subspace is that of Example 10. Now it becomes apparent that Algorithm 1 is computationally significantly more intensive than the method of Section 3.2. It took an hour to find a solution to the problem with $\epsilon=0.1$ on a modern workstation, after 274 iterations. Then $\kappa(V) \approx 1.3 \cdot 10^{5}$.

6. Conclusions. Conditioning of a nonsingular matrix subspace has been addressed in terms of matrix intersection (and respective nearness) problems. In the Frobenius norm, two computationally feasible approaches have been investigated. One leads to a differential equation on the unitary matrices and the other to a problem in computational algebraic geometry. For large and sparse problems, the latter alternative appears more attractive. Numerical experiments were carried out in the two dimensional case. 
[1] D.J. Bates, J.D. Hauenstein, A. J. Sommese and and C. W. Wampler, Bertini: Software for Numerical Algebraic Geometry, Available at http://www.nd.edu/ sommese/bertini.

[2] R. Bhatia, Matrix Analysis, Graduate Texts in Mathematics, 169. Springer-Verlag, New York, 1997.

[3] R. Bhatia, Positive Definite Matrices. Princeton Series in Applied Mathematics. Princeton University Press, Princeton, NJ, 2007.

[4] M. Byckling and M. Huhtanen, Approximate factoring of the inverse, Numer. Math., 117 (2011), pp. 507-528.

[5] D. Cox, J. Little and D. O'Shea, Using Algebraic Geometry, Graduate Texts in Mathematics, Vol. 185, Springer-Verlag, New York, 1998.

[6] L. Dieci, R. Russell, And E. VAn Vleck, Unitary integrators and applications to continuous orthonormalization techniques, SIAM J. Numer. Anal., 31 (1994), pp. 261-281.

[7] B. Eckmann, Hurwitz-Radon matrices revisited: from effective solution of the Hurwitz matrix equations to Bott periodicity, pp. 23-35, CRM Proc. Lecture Notes, 6, Amer. Math. Soc., Providence, RI, 1994.

[8] G.H. Golub and C.F. van Loan, Matrix Computations, The John Hopkins University Press, Baltimore and London, the 3rd ed., 1996.

[9] J. Goldstein And M. Levy, Linear algebra and quantum chemistry, Amer. Math. Monthly, 98 (1991), no. 8, pp. 710-718.

[10] E. Hairer and G. Wanner, Solving ordinary differential equations. II. Stiff and differentialalgebraic problems, 2. ed., Springer Series in Computational Mathematics, 14. SpringerVerlag, Berlin, 1996.

[11] E. Hairer, C. Lubich And G. Wanner, Geometric Numerical Integration. Structure-preserving algorithms for ordinary differential equations, 2. ed., Springer-Verlag, Berlin, 2006.

[12] P. Halmos, Bad products of good matrices, Linear and Multilinear Algebra, 29 (1991), no. 1, pp. $1-20$.

[13] R.A. Horn And C.R. Johnson, Matrix Analysis, Cambridge Univ. Press, Cambridge, 1987.

[14] M. Huhtanen, Factoring matrices into the product of two matrices, BIT, 47 (2007), pp. $793-$ 808.

[15] M. Huhtanen, Matrix subspaces and determinantal hypersurfaces, Ark. Mat., 48 (2010), pp. $57-77$.

[16] M. Huhtanen, Differential geometry of matrix inversion, Math. Scand., 107 (2010), pp. $267-$ 284.

[17] A. HuRwitz, Über die Komposition der quadratischen Formen, Math. Ann., 88 (1923), pp. 1-25

[18] J. Levin, Mathematical models for determining the intersections of quadric surfaces, Comput. Graphics Image Process., 11 (1979), pp. 73-87.

[19] P.-O. LöWDIN, On the non-orthogonality problem connected with the use of atomic wave functions in the theory of molecules and crystals, J. Chem. Phys., 18 (1950), pp. 365-374.

[20] R. Mavaddat, Network Scattering Parameters (Advanced Series in Circuits \& Systems), World Scientific, Singapore, 1996.

[21] B. Parlett, The Symmetric Eigenvalue Problem, Classics in Applied Mathematics 20, SIAM, Philadelphia, 1997.

[22] P. Pedersen, M-F. Roy And A. Szpirglas, Counting real zeros in the multivariate case, Computational Algebraic Geometry (F. Eysette and A. Galliago, eds.) Birhhäuser, Boston 1993, pp. 203-244.

[23] M. Postnikov, Lectures in Geometry, Smooth Manifolds, Mir Publishers, Moscow, 1989.

[24] J. Radon, Lineare Scharen orthogonaler Matrizen, Abh. Sem. Hamburg, 1 (1922), pp. 1-14.

[25] Y. SAAD, Iterative Methods for Sparse Linear Systems, 2nd ed., SIAM, Philadelphia 2003.

[26] A. Zalewska-Mitura and J. Zemánek, The Gerschgorin discs under unitary similarity, Banach Center Publ., 38, Polish Acad. Sci., Warsaw, (1997), pp. 427-441. 